\title{
Secondary Reduction Algorithm Based on Grid and Curvature Method
}

\author{
Shigang WANG ${ }^{1, a}$, Yong YAN $^{1, b}$, Shufeng JIANG ${ }^{1, c}$ \\ ${ }^{1}$ School of Mechatronics Engineering, Qiqihar University, Qiqihar 161006, China \\ ahljwangsg@163.com, b185662045@qq.com, cjsf349@sina.com
}

Keywords: Data reduction; Grid method; Curvature; Secondary Streamlining

\begin{abstract}
In reverse engineering, the point cloud data processing is the key, it will directly affect the quality and efficiency of the subsequent reconstruction of the surface. However, the amount of data obtained by three-dimensional laser scanning is often larger and redundant, which will increase the difficulty of the surface reconstruction, therefore, the point cloud data is needed to streamline. Depth analysis of the efficiency and accuracy of curvature grid method, a secondary streamlined algorithm is proposed, which take use of non-uniform grid streamline law based on octree to streamline to the point cloud, and re-use curvature streamline method based on B-spline surface fitting to streamline it. This method, whose streamline ratio is relatively high, can effectively retain the feature point cloud data, providing a great convenience for the follow-up of reverse engineering.
\end{abstract}

\section{Introduction}

With the rapid development of measurement technology, measuring efficiency and accuracy have been improved significantly. The large and dense point cloud can quickly obtained by scanning measurement for parts. When model is reconstructed, the direct use of these non-processed point cloud data to surface reconstruction is almost impossible. Many data points can cause a lot of problems [1-4], for example: to make the efficiency of a computer operated decrease, the greater storage space is needed, will consume a lot of time to generate surface, and so on. In addition, too many data points will also affect the smoothness of surface reconstructed, but the smoothness is very important in product design. On the basis of retaining product features point to reflect, at the same time removing a large amount of redundant data, it will help to shorten the modeling time and help to improve the modeling quality. Thus, in reverse engineering [5], to streamline the measured data is a key technology. In recent years, foreign scholars have done a lot of research for point cloud data reduction technique domestic and [6-8], and made a lot of algorithms on point cloud data reduction.

\section{Secondary Reduction Algorithm Based on Grid and Curvature Method}

Streamline Algorithm Based on Non-uniform Grid Octree. First, the algorithm uses octree non-uniform grid method to streamline the raw point cloud data. Octree space for segmentation model is a piece of data points surrounding the cloud and is sort of cube space. The first is to construct a minimum bounding streamline point cloud cube and put it as the root of the octree structure and then split into eight sub-cubes of uniform size, as shown in Figure 1. Then each child cube may be deemed to be the root of the child nodes, thus the number of sub-cube on a power of 2 . This division is called a uniform grid subdivision, which has been widely used in computer graphics and image processing. Octree structure in each cube has a node, and each cube contains eight sub-cubes, because it is broken down in the uniform, then on the $\mathrm{x}, \mathrm{y}, \mathrm{z}$ direction of each sub-cube is half of the side on the upper level, so a cube subdivided into eight sub-cubes, the cube is same on the same segmentation.

The principle of octree tree to streamline point cloud data is as follows: To establish a cube and involve all the raw point cloud data. In the structure of octree tree, the cube is considered as the root node, then divided into eight sub-cubes of uniform size, and each child is considered a child of the root node, divided down by this regular pattern, finally bounding box is divided into many small cubes. The divided principle used in this paper is that: if the child cube contains a 
point, the cube is called "blackbody"; if the child cube does not contain a point, the cube known as "white body". For a blackbody, if the number of points it contains more than $N$ ( $N$ is the set threshold, the paper $N$ is taken as 10), it will need to continue its subdivision until all points contained in the sub-cube less than $N$, then stop the subdivision.
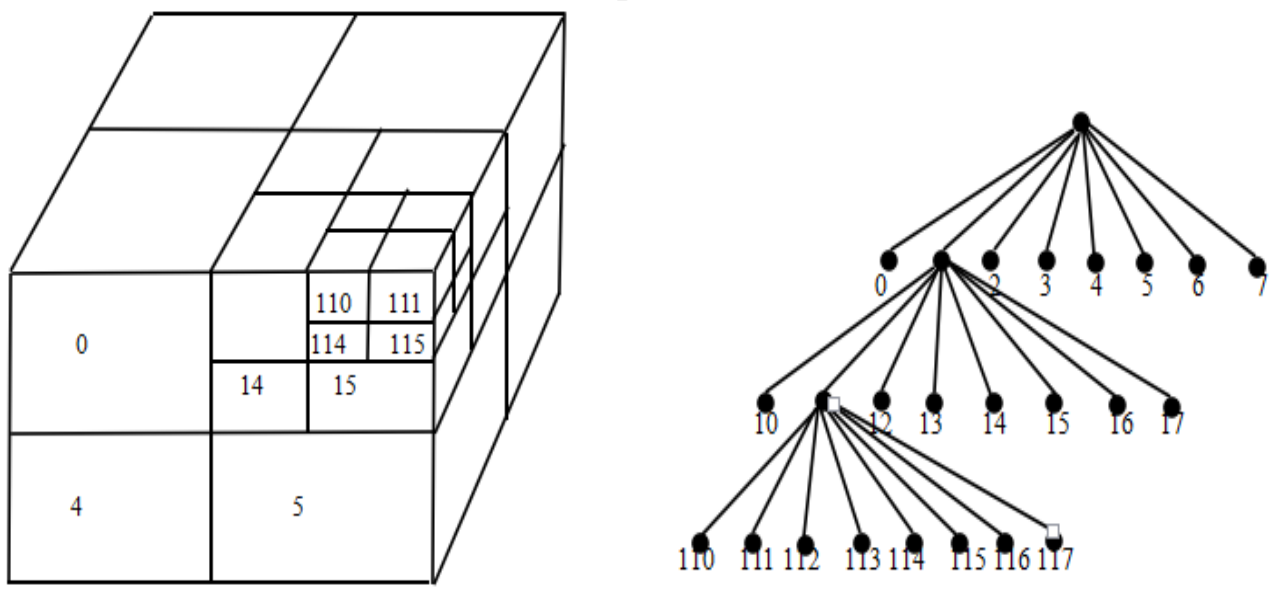

Fig. 1 Octree structure

When the division is completed, you need to select a point to instead the remaining points of the sub-cube. Currently there are several programs: (1) Selection of the nearest center of the cube; (2) Select the set of data points closest to the cube center point; (3) Select the set of data points of the cube center.

The first two methods select the original data points, so there is no need to consider the error before and after the error streamline, yet the third method generate their own point, so there is an error with the original data. In this paper, the second method is selected, which is closest to the cube set of data points in the center of gravity points, compared with the first method, it is more precise.

Streamline Curvature Surface Fitting Based on B-spline. After point cloud data is streamlined by non-uniform grid algorithm and it also need to further streamline by curvature algorithm. The first step is to calculate the curvature of the point cloud. The curvature is can not be calculated if calculated only from scattered data points, you can select the local data points for surface fitting, then the curvature of the surface can be solved according to equation fitting.

To calculate the curvature of a discrete point, we must first establish local coordinates, as is shown in Figure 2.

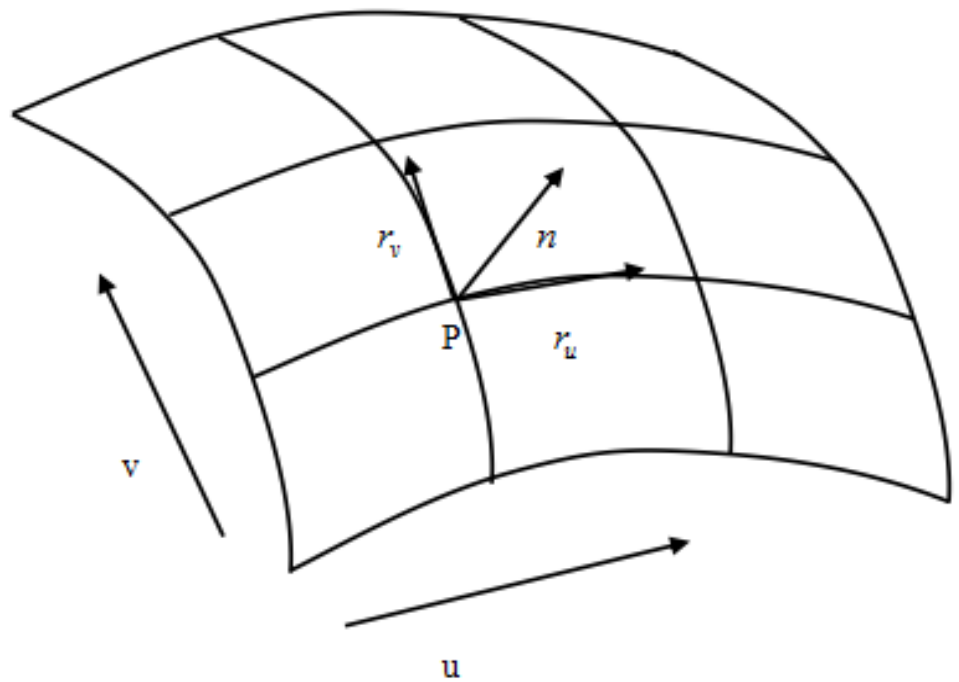

Fig. 2 Local coordinate of point $P$

Point $P$ is a point in the discrete points, $n$ is the unit normal vector of the point $P, r_{u}$ is tangent to the point $P$ in the direction of $u, r_{v}$ is tangent in the direction of $v$, So the local coordinate system of 
surfaces is constituted. Using this coordinate system, the surface in this area can be expressed as quadratic surface, the equation is $N(u, v)=(u, v, s(u, v))$, and $s(u, v)=a u^{2}+b u v+c v^{2} a, b, c$ are coefficients to be determined. $s_{u}, s_{v}, s_{u v}, s_{u u}, s_{v u}, s_{v v}$ are respectively as a function of $s$ with respect to the first-order and second-order with $u$ and $v$. The unit normal vector $n$ of the surface is that:

$$
n=\frac{s_{u} \times s_{v}}{\left\|s_{u} \times s_{v}\right\|}
$$

According to the surface of the first basic form:

$$
E=s_{u} \cdot s_{u}, F=s_{u} \cdot s_{v}, G=s_{v}, s_{v}
$$

According to the surface of the second basic form:

$$
L=s_{u u} \cdot n, M=s_{u v} \cdot n, N=s_{v v} \cdot n
$$

Gaussian curvature of the surface can be obtained:

Mean curvature:

$$
K=\frac{L N-M^{2}}{E G-F^{2}}
$$

By the formula:

$$
H=\frac{E N-2 F M+G L}{2\left(E G-F^{2}\right)}
$$

$$
K_{1,2}=H \pm \sqrt{H^{2}-k}
$$

You can calculate the main curvatures $K_{1}$ and $K_{2}$. This paper selects the mean curvature $H$ for streamlining data points.

Step 1: Calculate the mean of mean curvature of all local point set;

Step 2: Compared with the average curvature point $H$ and $H_{\mu}$, divide the point set into two subsets, when $H \leq H_{\mu}$, then put point set into the child set of $P_{1}$, when $H>H_{\mu}$, then put point set into the child set of $P_{2}$;

Step 3: For a subset of $P_{1}$ and $P_{2}$, streamline them respectively using the rate of $\varepsilon_{1}$ and $\varepsilon_{2}$, $\varepsilon_{1}$ and $\varepsilon_{2}$ are as custom thresholds.

After the above steps are completed, the curvature of the point cloud data is completed. The algorithm not only preserves the characteristic details of the original point cloud but also retains the appropriate point in the large curvature region in the point cloud, and cut more points in the curvature of the smaller area.

\section{Streamline Experiments and Analysis}

In order to verify the validity of the second streamline algorithm proposed in this paper, water bottles and shoes' data points were streamlined, and the experimental results were deeply analyzed. Evaluate the advantages and disadvantages of the algorithm through graphic visual effects, that is, on a qualitative evaluation.

Streamline Experimental on Bottle Model. This section uses different kinds of measurement data algorithms to process and streamline the bottle model. Compare the streamline algorithm using control variable method approach, depending on the application of the bottle, as well as the requirements of its accuracy, to control the rate of streamline point cloud model at $75 \%$, then evaluate the algorithm through the show up after the effect of streamlining plans by different algorithms. Figure 3 (a) is the original point cloud data, a total of 95,516 data points. Figure 3 (b) is point cloud data streamlined by the bounding box method, the image shows that the deletion of data points using uniform algorithm, which point cut in the flat area are favorable, but the ability to capture the changes 
in the characteristics of the region point is poor. Figure 3 (c) is the point cloud data streamlined via the angle sine law, As can be seen form the comparison of the diagram in Figure 3 (b), their ability of the algorithm to capture a point on the changes in the characteristics of the region improves, but show a shortage at the point of processing flat area, more confusion. Figure 3 (d) is point cloud data processed and streamlined by the second reduction algorithm, the difference can be seen more clearly between in Figure 3 (b) and Figure 3 (c), the larger curvature point is reserved more, that is the point of bottom, bottleneck, bottle area is reserved more; Little change in curvature point is moderate and uniform cut, that is the point of bottle region has been pruned more and uniform.

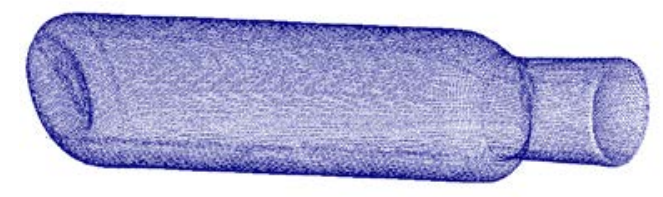

(a) The original of water bottle

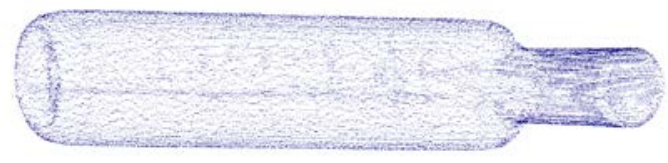

(c) Bottle angle sine data reduction

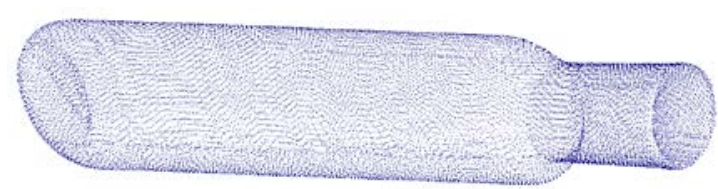

(b) Water bottle bounding box data reduction

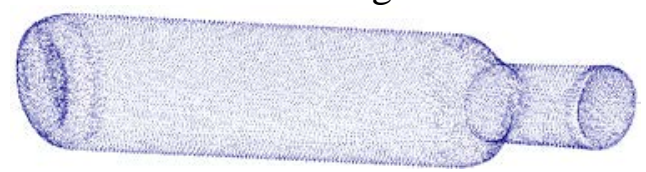

(d) Water bottle data secondary streamline

Fig. 3 Streamline experimental on bottle model

Streamline Experimental Shoe Models. According to the actual application of the shoe, and its accuracy, to control the rate of streamline point cloud model shoe in $65 \%$, then compare the difference of reduction algorithm. Figure 4 (a) is the original point cloud data, a total of 26,383 data points. Figure 4 (b) is the point surrounded by streamlined data box, we can see that data reduction is more uniform, but the ability to capture the feature point is not strong. Figure 4 (c) is the point data streamlined by sine of the angle, compared with the previous diagram can be obtained that the ability to capture the feature point has increased, but the point processing flat region still seems complicated uneven. Figure 4 (d) is data point streamlined by the second reduction algorithm, compared with the former two figures, as can be seen, the feature point that is at the edge of the shoe to retain a greater amount of points, in a flat area of point-shoe language point is relatively less reserved, more streamlined uniform.

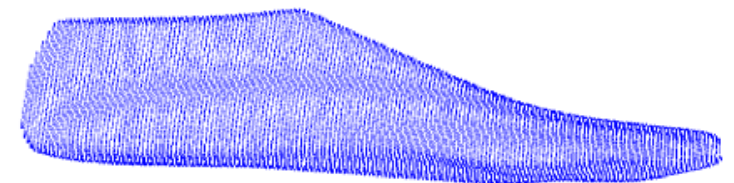

(a) The original point cloud data of the shoe

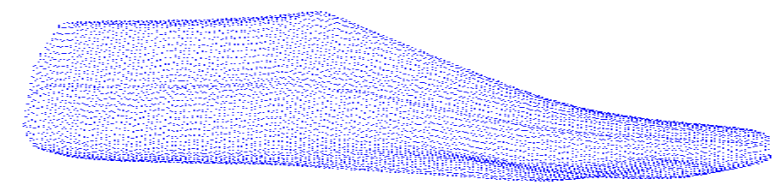

(c) shoe angle sine data reduction

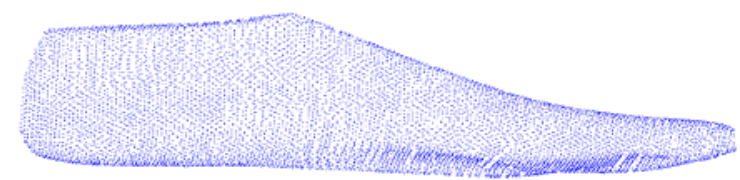

(b) Shoes bounding box data reduction

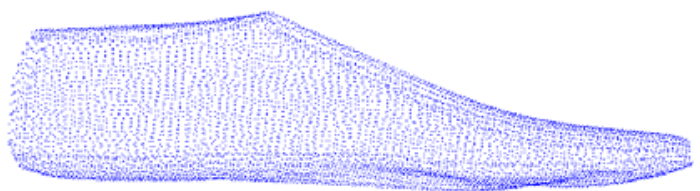

(d) secondary data reduction of the shoe

Fig. 4 Shoe point cloud data reduction results

\section{Conclusion}

In reverse engineering, to measure model using laser measuring equipment will get a huge amount of data, which increases the difficulty of subsequent modeling, it is necessary to streamline the processing point cloud data. In this paper, we proposed a secondary reduction algorithm, the new algorithm combines the simplicity and accuracy of the grid method streamlined and streamlines curvature. First, streamline the raw point cloud data by using octree non-uniform grid algorithm, and 
then do the second reduction by using B-spline surface fitting algorithm. The first aim to streamline is to retain key feature points and minimize the point cloud; the second purpose is to delete the redundant feature points appropriately. Finally, experimental demonstration for different reduction algorithm, the results show that, in the same circumstances of leaner than, the proposed algorithm compared with other algorithms, Which can retain data points with precisely detailed features of the original, and the point is relatively flat area evenly cut to facilitate the subsequent surface reconstruction.

\section{Acknowledgements}

This work was supported by Project of Department of Education of Heilongjiang Province, China (Grant No. 12521597) and Qiqihar University youth teacher research startup project (Grant No. 2014k-M04) and Qiqihar University Graduate Innovation Research Project (Grant No. YJSCX2014-025X).

\section{References}

[1] R R Martin, I A Stroud, and A D Marshal. Data reduction for reverse engineering [C]. Computer and Automation Institute of Hungarian Academy of Science, January 2010 (1068): 63-69.

[2] Veron P, Leon J L. Static polyhedron simplification using error measurements [J]. Computer-Aided Design, 201229 (4): 187-296.

[3] Chen Y H, Neg C T, Wang Y Z. Data reduction in integrated reverse engineering and rapid prototyping [J]. The International Journal of Computer Integrated Manufacturing, 200712 (2): 97-103.

[4] K. H. Lee, H. Woa and T. Suk. Data reduction methods for reverse engineering [J]. The International Journal of Advanced Manufacturing Technology, 2001 (17): 735-743.

[5] Bin Yang, Changshuang Dong. Reverse engineering was applied in the actual production [J]. Mechanical Engineering and Automation, 2011 (5): 174-175.

[6] Pingjiang Wang, Xuemei Huang, Jihong Chen. Triangular piece intensive laser measuring surface approximation method for three-dimensional data [J]. Journal of Engineering Graphics, 1998 (l): 17-28.

[7] Xiaodong Tian, Guirong Shi, Xueyu Fu. Reverse engineering of complex surface kind of its key technologies [J]. Mechanical Design and Manufacturing Engineering, 200029 (4): 1-4.

[8] Hua Yan, Julin Guang, Shuhuang Cai. Automotive reverse engineering exploration cover a number of key technologies [J], Die and Mould Technology, 2001 (2): 4-8. 